\title{
A Review on Immunomodulatory Effects of Plant Extracts
}

\author{
Rama Bhat P* \\ Dept of PG Studies and Research in Biotechnology, Alva's College, Moodbidtri- 574 227, \\ Karnataka, India
}

*Correspopnding author: Rama Bhat P, Dept of PG Studies and Research in

Review Article

Volume 2 Issue 6

Received Date: April 28, 2018

Published Date: May 07, 2018

Biotechnology, Alva’s College, Moodbidtri- 574 227, Karnataka, India, Email: bhat_pr@rediffmail.com

\begin{abstract}
India is the mother land of Ayurveda and herbal plants. Numbers of plants identified from wild have one or the other medicinal properties which were used in traditional and folklore practices from ancient time are scientifically proved now. All the plants contain secondary metabolites or active compounds which will alter or trigger the body immune system and leading to fight against invaders. So herbal extracts will functions as immunomodulatory, suppressive or stimulants in our body.
\end{abstract}

Keywords: Immunomodulatory; Immunosuppressive; Immune system; Plant Extract; Medicinal Plants

\section{Introduction}

Immunology is one of the most rapidly developing areas of medical biotechnology research and has great promises with regard to the prevention and treatment of a wide range of disorders such as inflammatory diseases of the skin, gut, respiratory tract, joints and central organs. In addition infectious diseases are now primarily considered immunological disorders while neoplastic diseases, organ transplantation and several autoimmune diseases may involve in an immunosuppressive state.

Modulation of immune system denotes to any change in the immune response that can involve induction, expression, amplification or inhibition of any part or phase of immune response. Stimulation of immune response is desired for certain people such as immunocompromised patients. Whereas suppression of immune response is thought for others, such as transplant recipient, patients with autoimmune diseases, allergic and inflammatory diseased patients.
India has a very rich diverse faunal and floral wealth spread across the length and breadth of country. Biodiversity hotspots like Himalayan region and Western Ghats are bestowed with innumerous number of potential medicinally important plants whose scientific research are yet to be taken up. Herbs and/or plants are the oldest friends of mankind. They not only provided food and shelter but also served to cure different ailments. Herbal medicine, sometimes called traditional or natural medicine, has always existed in one way or another in different cultures and civilizations, such as Ayurvedic (India), Egyptian, Western, Chinese, Kampo (Japan) and Greco-Arab or Unani- Tibb (south Asia). The medicinal properties of certain plants have been known for centuries. More than a quarter of the medicines in use today come from plants, i.e. from traditional medicine, currently, with the active encouragement of the WHO. Traditional medicine all over the world is currently being revalued through extensive research activity on various plant species and their therapeutic properties. According WHO, about three-quarters of the world population relies 
upon traditional remedies (mainly herbs) for the health care of its people.

\section{Immune System}

The immune system is one of our most complex biological systems in the body. The basic role of the immune system is to distinguish self from non-self. This non-self could be an infectious organism, a transplanted organ or an endogenous cell that can be mistaken as a foreign [1]. The immune responses of the human body against any non-self are of two types (a) innate (or natural or non-specific) and (b) adaptive (or acquired or specific) [2]. Both these responses have two components each, that is, cellular and humoral.

Innate immunity lacks specificity as there is no involvement of memory cells. Acquired immunity on the other hand is specifically adapted for inducing pathogens and response improves with subsequent exposures to the same pathogen due to the presence of memory cell line. In the innate cellular immunity there is involvement of monocytes-macrophage system, while in innate humoral immunity there is activation of component system. On the other hand the cellular components of acquired immunity consist of T-lymphocytes, while the humeral components of this system involve the role of B-lymphocytes. Normally in innate and acquired immune responses act in concerted manner to contain or eradicate infection. In some cases innate responses are enough to neutralize the offending agent. However, in many other cases, certain service of innate immune system, such as antigen presenting cells (APC), can also process the offending agents in this smaller fragment which then activate adaptive immune system to neutralize or kill these pathogens [3].

The elements formed in the blood are erythrocytes (RBC), leukocytes (WBC) and thrombocytes (platelets). The leukocytes are of two types: granulocytes (neutrophils, eosinophils, and basophils) and agranulocytes (T-lymphocytes, B-lymphocytes and bonocytes). The process by which blood cells are formed is called haemopoiesis. All such cells are involved in exerting immune response develops from pluripotent haemotopoietic stem cells which resides in bone marrow. These stem cells gives rise to lymphoid stem cells trilineage myeloid stem cells, megakaryocytes (form platelets) and erythroblasts (form erythrocytes). The lymphoid stem cells through their progenitors give rise to mature lymphocytes (T-lymphocytes and B-lymphocytes) and natural killer cells (NK cells) [4]. T-lymphocytes and B-lymphocytes are involved in mediating adaptive immune responses while NK cells exert innate immune response along with mature cells originating from trilineage myeloid stem cells. When exposed to specific antigens, B-lymphocytes differentiate into antibody producing plasma cells in bone marrow. Simultaneously T-lymphocytes under the influence of thymic hormones, migrate to the thymus and on appropriate stimulus by antigen presenting cells acquire T-cell receptor (TCR) and get differentiated into helper $\mathrm{T}$ - cell (with specific protein cluster of differentiation-CD4+) and cytotoxic T-cell (with specific protein cluster of differentiation-CD8+). The CD4+(TH cell) subtypes of $\mathrm{T}$ cells differentiate further outside the thymus into several phenotypes: TH1, TH2 and TH3 which are distinguished by the different cytokines (IL-2 and IFN- $\gamma$ ) they synthesize.TH1 T cells produce cytokines that stimulate proliferation and differentiation of $\mathrm{T}$ lymphocytes and NK cells. These cytokine play an important role in cell mediated immunity. TH2 T cells release cytokine (IL-4, IL-5, IL-10 and IL-13) that stimulate B lymphocytes production for humoral immunity. TH3 T cell play an important role in resting faces of immune response and in the production of antiinflammatory immunoglobin-A (IgA) antibodies that are important in secretory immunity [5-9].

Immunomodulators: These are biological or synthetic substances that can stimulate suppress or modulate any aspect of the immune system including both adaptive and innate arms of the immune system.

\section{Classification of Immunomodulators}

Clinically, immunomodulators can be classified into the following three categories:

Immunoadjuvants are used to enhance the efficacy of vaccines and therefore could be considered specific immune stimulants. Immunoadjuvants hold the promise of being the true modulators of the immune response. It has been proposed that they be exploited as selectors between cellular and humoral helper T1 (Th1) and helper T2 cells (Th2), immunoprotective, immunodestructive, and reagenic (immunoglobulin E (IgE) versus IgG type immune responses-posing a real challenge to vaccine designers.

Immunostimulants are inherently non-specific as they are envisaged as enhancements to a body's resistance to infection. They can act through innate as well as adaptive immune responses. In healthy individuals, the immunostimulants are expected to serve as prophylactic and promoter agents, i.e., as immunopotentiators, by enhancing the basic level of immune response. In the individual with impairment of immune response, they are 
expected to act as immunotherapeutic agents. Immunosuppressants are a structurally and functionally heterogeneous group of drugs, which are often concomitantly administered in combination regiments to treat various types of organ transplant rejection and autoimmune diseases.

\section{Plant Extracts as Immunomodulators}

Different plant extracts in various solvent systems employed by various people to study the immunomodulatory effects on rat and mice as model organism at different dosages with different experimental parameters. The result also varies according to the plant extracts. Increased delayed hypersensitivity titre [10-18], decreased hemagglutination [10-13,15-19], spleen lymphocyte proliferation and increased natural killer cell cytotoxicity [19], changes in delayed type hypersensitivity and also increased the haemagglutination titre which indicates that both humoral and cell mediated response [15-16,20-21].

Significantly enhanced the phagocytic activity of peritoneal macrophages in vivo $[11,12,19,22]$, extract did not show much change in the body or tumor weight but showed lower tumor growth. Higher concentrations appeared to show more effective inhibition, increased WBC count, decrease in cell mediated and humoral immunity $[11,15,16,19,22]$.

Some plant extracts are immunosuppressive some may immunomodulatory $\quad[12-13,15-16,19,21], \quad$ exhibit significant increase in foot pad thickness/ paw value [13,15-17], bone marrow cells, $\alpha$-esterase cells and immunoglobulin levels indicating the immunomodulatory effect of the extract $[17,23]$.

Kumar et al. [24] made a review on the immunomodulatory effects of different parts of 8 medicinal plants with respect to their active compounds which are responsible for their immunostimulant and immunosuppressive activity [24]. Previously Smith et al. [25] narrated the immunomodulatory activity of 21 plant species with different parts of such plants used (Table 1). So immense information of such plants from India with various active components useful in curing various diseases [26,27].

\begin{tabular}{|c|c|c|c|}
\hline Sl No. & Plant name & Family & Part/s use \\
\hline 1 & Boerhaavia diffussa & Nyctaginaceae & Root \\
\hline 2 & Curcuma longa & Zingiberaceae & Leaf \\
\hline 3 & Rhododendron spiciferum & Ericaceae & Whole plant \\
\hline 4 & Caesalpinia bonducella & Caesalpiniaceae & Whole plant \\
\hline 5 & Tinospora cordifolia & Menispermaceae & Whole plant \\
\hline 6 & Capparis zeylanica & Capparidaceae & Shole plant \\
\hline 7 & Luffa cylindrical & Cucurbitaceae & Root \\
\hline 8 & Withania somnifera & Solanaceae & Root \\
\hline 9 & Asparagus racemosus & Asparagaceae & Leaf \\
\hline 10 & Panax ginsengs & Araliaceae & Floed \\
\hline 11 & Nelumbo nucifera & Nymphaeaceae & Flower \\
\hline 12 & Azadiracta indica & Meliaceae & Compositae \\
\hline 13 & Arnica montena & Asteraceae & Latex \\
\hline 14 & Calendula officinalis & Asteraceae & Leaf \\
\hline 15 & Echinacea purpurea & Euphorbiaceae & Lamiaceae \\
\hline 16 & Euphorbia tirucalli & Ocimum sanctum &
\end{tabular}

Table 1: List of plants having immunomodulatory activity [26].

In one of the study with cod liver oil which showed an increase in specific immunity against an invading pathogen, increased antibody titre value and phagocytic index and in low dose increases humoral immunity [28].
Mechanisms including the blood brain barrier (BBB) integrity, oxidative stress, chronic stress, hippocampal neurogenesis, microglial activation and chronic low-grade neuroinflammation, have shown to be related to cognitive 


\section{Virology \& Immunology Journal}

changes across age. Supplementation with one or more plant based extracts or nutraceuticals that act on these mechanisms may be an important next step toward preventing age associated cognitive decline. In recent years there has been a growing interest in exploring naturally forming compounds on protecting BBB permeability, ameliorating microglial activation and/or neuroinflammation, with the aim to develop treatments to prevent cognitive decline. Various plant based extracts have shown to exert protective effects on the BBB by preserving BBB integrity and function. These important compounds are found in plants (e.g., Ginkgo biloba, Panax ginseng, and Bacopa monnieri) and food sources (e.g., resveratrol, tea polyphenols, plant alkaloids and antioxidants). Since microglial cells contribute to neurodegenerative diseases by activating neuroinflammatory processes and oxidative stress, natural compounds that supress these mechanisms may be key therapies in preventing cognitive decline in older individuals. Potential antioxidant therapies are phytochemicals including curcumin, EGCG and resveratrol which interestingly also play a role on microglial cells [29].

However, the translational gap between in vitro, in vivo and clinical studies is still a major issue and there is a paucity of studies looking at the immunomodulatory effects of the discussed plant extracts and secondary plant metabolites in a healthy population. Moreover, careful consideration should be made in respect to the immunosuppressive and immunomodulatory effects of the plant extracts and active plant compounds, as for instance hypo-activity of microglial cells has likewise demonstrated to be involved in disturbing normal brain functioning [30]. Therefore, the long-term effects of the herbal treatments should be studied more extensively.

Another aspect to take into consideration is that humans present a broad range of responses to similar plant based extracts related to genetic and epigenetic modulations involved in the metabolism and distribution of the active compounds [31]. Interestingly, modern science is investigating the effects of many traditionally used medicinal plant extracts and plant compounds and should perhaps also evaluate the great potential of other fundamental principles underlying traditional medicinal systems [25].

Modulation of the immune response through stimulation or suppression may help in maintaining a disease-free state. Agents that activate host defense mechanisms in the presence of an impaired immune responsiveness can provide supportive therapy to conventional chemotherapy Immunostimulation in a drug-induced immunosuppression and immunosuppression in an experimental hyper reactivity model by the same preparation can be said to be true immunomodulation. The presence of immunostimulant compounds in higher plants has been extensively reviewed but only a limited amount of immunosuppressive products of plant origin have been delayed reported. Such products, if well tolerated by the patient may be developed into alternative coadjuvants in the treatment of disorders caused by an exaggerated or unwanted immune response, such as in autoimmune diseases, allergies, glomerulonephritis, chronic hepatitis etc.

One of the earliest immune response can be seen and measured by studying the hematological parameters of an animal. Accordingly leukocyte counts were measured. Blood cells are the first invading non self materials cells to be responding to invading non self materials. An immunomodulatory effect of any immune substance would first see as a change in leukocyte count. There was a significant increase in the leukocyte count by administrating the extract.

Hemoglobin is also one of the important parameter that would reveal the health status of the individual. But no significant change was seen in the study, but there was a significant increase in bone marrow cellularity and $\alpha$ esterase positive cells.

The haemagglutination antibody titre was used to assess humoral immune response. Antibody molecules, a product of B lymphocytes and plasma cells are central to humoral immune responses At the selected dose antibody titre were observed in rats treated with aqueous extracts of seed of Myristica fragrans The augmentation of the humoral immune response to SRBCs by extract evidenced by increase in the antibody titres in the blood of rats [17].

Bharathan et al. investigated the immunomodulatory effect of seed extract of $M$. fragrans. The SRBC-induced delayed-type hypersensitivity was used to assess the effect of the fraction on cell-mediated immunity [17]. Cellmediated immunity (CMI) involves effector mechanisms carried out immunity (CMI) involves effector mechanisms carried out responses are critical to defense against infectious organisms, infection of foreign grafts, tumor immunity and delayed-type hypersensitivity reactions Therefore, increase in DTH reaction in rats response to $\mathrm{T}$ 
cell dependent antigen revealed the stimulatory effect of aqueous extracts of seed of Myristica fragrans on T- cells.

One fundamental principle in Ayurveda, the Indian traditional medicinal system, is the prakriti, which defines a person's true nature based on psychosomatic features [32]. The prakriti is independent of racial, ethnic and geographical factors and is highly correlated with molecular and genetic profiles [33-35]. Therefore, the future research could be focused on personalized herbal supplementation to prevent age-related cognitive decline and thus aim for an optimal response through a personalized rebalance of the various underlying mechanisms. Furthermore, some of the common cofounding factors that currently debilitate comparisons within and between researches could be rectified. In addition, the prakriti is likely also underlying the discrepancies found between other study outcomes as either increased or decreased amounts of proinflammatory cytokines in similar research settings, because the homeostatic imbalance can take a different course depending on someone's prakriti [35]. So lots of plants have immunomodulatory effect which were found distributed in India have been practically studied [27].

\section{Conclusion}

A drug, as per the science of herbal drug can result in different action based on the concentration of the drug prescribed. It is very common in Ayurvedic practices were concentration dependent effect is achieved as per the requirement. Therefore, there is every possibility that an extract might trigger a toxicological or a negative effect on any of the physiological or immunological system of the body. Nevertheless the drug tested has evidently shown the immunomodulatory effect when used at lower concentration. As a concludery mark could be employed to boost immune system at low concentration and it should also be kept in mind that high concentration of same could lead to physiological effect in the body. The extract contain several secondary metabolite fractions will induce immune system effectively.

\section{References}

1. Sharma P (1983) Charaka Samhita, Chikitasasthana, Chaukhamba Orientalia. Varanasi, India pp: 54.

2. Sainis KB, Sumariwalla PF, Goel A, Chintalwar GJ, Sipahimalani AT, et al. (1997) Immunomodulatory Properties of Stem Extracts of Tinospora cordifolia:
Cell Targets and Active Principles. Narosa Publishing House pp: 155.

3. Thatte UM, Dhanukar SA (1986) Ayurveda and contemporary scientific thought. Trends in Pharmacological Sci 7: 247-251.

4. Katiyar CK, Brindavanam NB, Tiwari P, Narayana DBA (1997) Immunomodulation (Eds.) Narosa Publishing House Pp: 163-187.

5. Wei J, Xu H, Davies JL, Hemmings GP (1992) Increase of plasma IL-6 concentration with age in healthy subjects. Life Sci 51(25): 1953-1956.

6. Roubenoff R, Harris TB, Abad LW, Wilson PW, Dallal GE, et al. (1998) Monocyte cytokine production in an elderly population: effect of age and inflammation. J Gerontol A Biol Sci Med Sci 53(1): M20-M26.

7. Forsey RJ, Thompson JM, Ernerudh J, Hurst TL, Strindhall J, et al. (2003) Plasma cytokine profiles in elderly humans. Mech Ageing Dev 124(4): 487-493.

8. Stowe RP, Peek MK, Cutchin MP, Goodwin JS (2010) Plasma cytokine levels in a population-based study: relation to age and ethnicity. J Gerontol A Biol Sci Med Sci 65(4): 429-433.

9. Álvarez-Rodríguez L, López-Hoyos M, Muñoz-Cacho P, Martínez-Taboada VM (2012) Aging is associated with circulating cytokine dysregulation. Cell Immunol 273(2): 124-132.

10. Fulzele SV, Satturwar PM, Joshi SB, Dorle AK (2003) Study of the immunomodulatory activity of Haridradi Ghrita in rats. Indian J Pharmacol 35(1): 51-54.

11. Shah AS, Wakade AS, Juvekar AR (2008) Immunomodulatory activity of methanolic Extract of Murraya koenigii (L) Spreng. leaves. Indian J Experimental Biol 46(7): 505-509.

12. Agarwal SS, Saurabh C, Khadase SC, Talele GS (2010) Studies on immonomodulatory activity of Capparis zeylanica leaf extract. International J Pharmaceutical Sciences and Nanotechnology 3(1): 887-892.

13. Patil VV, Bhangale SC, Patil VR (2010) Studies on immunomodulatory activity of Ficus carcia, International Journal of Pharmacy and Pharmaceutical Sciences 2(4): 97-99. 


\section{Virology \& Immunology Journal}

14. Singh S, Yadav CPS, Noolvi MN (2012) Immunomodulatory activity of butanol fraction of Gentiana olivieri Griseb. on Balb/C mice. Asian Pacific J Tropical Biomedicine 2(6): 433-437.

15. Ballal SR, Bhat PR, Sumalatha, Acharya S (2012) Studies on immunosti mulatory effect of Pajanelia longifolia (Willd.) Schumann on albino rats. Internatl J Res Pharmaceutical Biomedical Sci 3(4): 1642-1651.

16. Sumalatha, Bhat PR, Ballal SR, Acharya S (2012) Studies on immunomodulatory effects of Salacia chinensis L. on albino rats. J Applied Pharmaceutical Sci 2(9): 98-107.

17. Bharathan D, Bhat PR, Prajna PS, Jayadev K, Katte Basava (2013) Immuno-modulatory effect of seed extracts of Myristica fragrans Houtt. on Albino rats. Agriculture Sci Res J 3(10): 324-332.

18. Patila PR, Patil MR, Manea A, Patila S (2013) Immunomodulatory effects of fruits of Barringtonia racemosa Linn. Internatl J Basic Clinical Pharmacol 2(2): 216-219.

19. Gaur K, Kori ML, Nema RK (2009) Comparative screening of immunomodulatory activity of hydroalcoholic extract of Hibiscus rosa sinensis Linn. and ethanolic extract of Cleome gynandra Linn. Global J Pharmacol 3(2): 85-89.

20. Chandu AN, Kumar CS, Bhattacharjee C, Debnath SK, Kannan KK (2011) Studies on immunomodulatory activity of Aloe vera. Internatl J Applied Biol Pharmaceutical Technol 2(1): 19-22.

21. Choudhary GP (2012) Immunomodulatory activity of alcoholic extract of Terminalia belerica Linn. in mice. Der Pharmacia Lett 4 (2): 414-417.

22. Lu Y, Fan J, Zhao Y, Chen S, Zheng X, et al. (2007) Immunomodulatory activity of aqueous extract of Actinidia macrosperma. Asia Pacific J Clinical Nutrit 16(1): 261-265.

23. Vaibhav DA, Arunkumar W (2010) Pharmacological study of Tinospora cordifolia as an immunomodulator. Internatl J Current Pharmaceutical Res 2(4): 52-54.

24. Kumar D, Arya V, Kaur R, Bhat ZA, Gupta VK, et al. (2012) A review of immunomodulators in the Indian traditional health care system. J Microbiol Immunol Infection 45(3): 165-184.
25. Smith HR, Heusel JW, Mehta IK, Kim S, Dorner BG, et al. (2002) Recognition of a virus-encoded ligand by a natural killer cell activation receptor. Proc Natl Acad Sci 99(13): 8826-8831.

26. Kumar SV, Sharma SP, Rupesh D, Nitin K (2011) Immunomodulatory effects of some traditional medicinal plants. J Chem Pharm Res 3(1): 675-684.

27. Gulati K, Ray A, Debnath PK, Bhattacharya SK (2002) Immunomodulatory studies on Indian medicinal plants. J Natural Remedies 2: 121-131.

28. Asad M, Srivathsa B (2012) Immunomodulatory activity of cod liver oil. Iranian J Pharmacol Therapeutics 11(1): 20-25.

29. Kure C, Timmer J, Stough C (2017) The Immunomodulatory effects of olant extracts and plant secondary metabolites on chronic neuroinflammation and cognitive aging: A mechanistic and empirical review. Front Pharmacol 8: 117.

30. Niraula A, Sheridan JF, Godbout JP (2017) Microglia priming with aging and stress. Neuropsychopharmacol 42(1): 318-333.

31. Szarc vel Szic K, Declerck K, Vidaković M, Vanden Berghe W (2015) From inflammaging to healthy aging by dietary lifestyle choices: is epigenetics the key to personalized nutrition? Clin Epigenetics 7: 33.

32. Prasher B, Varma B, Kumar A, Khuntia BK, Pandey R, et al. (2017) Ayurgenomics for stratified medicine: TRISUTRA consortium initiative across ethnically and geographically diverse Indian populations. J Ethnopharmacol 197: 274-293.

33. Joshi K, Ghodke Y, Shintre P (2010) Traditional medicine and genomics. J Ayurveda Integr Med 1(1): 26-32.

34. Ghodke Y, Joshi K, Patwardhan B (2011) Traditional Medicine to modern pharmacogenomics: ayurveda prakriti type and CYP2C19 gene polymorphism associated with the metabolic variability. Evid Based Complement Alternat Med 2011: 249528.

35. Prasher B, Gibson G, Mukerji M (2016) Genomic insights into ayurvedic and western approaches to personalized medicine. J Genet 95: 209-228. 